\title{
Verantwortungszurechnung im EU- Mehrebenensystem während der Eurokrise: Wer kontrollierte die Troika-Institutionen?
}

\author{
Eugénia da Conceição-Heldt
}

Online publiziert: 2. März 2016

(c) Die Autor(en) 2016. Dieser Artikel ist auf Springerlink.com mit Open Access verfügbar.

Zusammenfassung Mit der Einrichtung der Troika-Institutionen hat die Frage der Kontrollmöglichkeiten und Verschränkung von Macht im EU-Mehrebenensystem an Bedeutung gewonnen. Vor diesem Hintergrund untersucht dieser Beitrag die Verantwortungszurechnung (accountability) der Troika-Institutionen während der Eurokrise. Dabei argumentiere ich, dass Delegations- und Treuhandmodi unterschiedlichen Logiken der Verantwortungszurechnung entsprechen, in denen die Kontrolle der Agenten oder Treuhänder durch die Prinzipale oder Begünstigten auf verschiedenen Ebenen stattfindet. Während im Delegationsmodus formale Kontrollmechanismen überwiegen, sind diese im Treuhandmodus informeller Art. Zur Illustration des Arguments vergleicht der Beitrag diese zwei Modi im Hinblick auf die darin verankerten Kontrollmechanismen: die Delegationsbeziehung zwischen der Kommission und dem Rat der Europäischen Union einerseits sowie die Treuhandbeziehung zwischen der Europäischen Zentralbank und den Eurostaaten andererseits.

\section{Accountability in the EU Multi-level System during the Euro Crisis: Who Controlled the Troika Institutions?}

\begin{abstract}
With the establishment of the troika institutions during the Euro crisis, issues related to possibilities for control and constraint of these institutions, as well as their accountability, became prominent. This contribution analyses the accountability of the troika institutions, that is, the extent to which agents and trustees
\end{abstract}

Prof. Dr. E. da Conceição-Heldt ( $₫)$

Institut für Politikwissenschaft,

Technische Universität Dresden,

Von-Gerber-Bau, Bergstr. 53, 01062 Dresden, Deutschland

E-Mail: eugenia.conceicao-heldt@tu-dresden.de 
are controlled at different levels by their principals or beneficiaries. Specifically, I distinguish between a delegation and a fiduciary mode of accountability in EU governance. While the delegation mode is characterised by formal hierarchical, supervisory and legal control mechanisms, the fiduciary mode is marked by informal peer organisations and public reputational mechanisms. In order to illustrate these two modes of accountability, this article examines a delegation relation (European Commission and Council of Ministers) and a fiduciary relation (European Central Bank and Eurozone-Member States) during the financial bailout assistance programmes.

\section{Einleitung ${ }^{1}$}

Mit der massiven Kritik an den Troika-Institutionen - zusammengesetzt aus Europäischer Kommission (nachfolgend „,die Kommission“), Europäischer Zentralbank (EZB) und Internationalem Währungsfonds (IWF) - hat die Thematik der demokratischen Kontrolle und der Verschränkung von Macht in der Europäischen Union (EU) an Bedeutung gewonnen. ${ }^{2}$ Im Rahmen der Einrichtung der Hilfsprogramme zur Lösung der Staatsschuldenkrise statteten die Eurostaaten diese drei Institutionen mit erheblichen Entscheidungskompetenzen aus. Die Eurostaaten ermächtigten sie dazu, die Umsetzung der Programme in den Krisenländern (Portugal, Griechenland, Irland und Zypern) zu überwachen. Auf der Grundlage der von den TroikaInstitutionen verfassten Berichte entschieden die Eurostaaten, ob die nächste Finanztranche an die Krisenländer überwiesen werden konnte. Die Ausstattung von nicht direkt gewählten Institutionen mit neuen Kompetenzen hat gleichzeitig Fragen der Verantwortungszurechnung (nachfolgend wird auch der englische Begriff „accountability“ verwendet) im EU-Mehrebenensystem aufgeworfen. Analog zu der schon lange andauernden Diskussion in der klassischen politikwissenschaftlichen Institutionenlehre über Gewaltenteilung, die von der Kontrolle, Verschränkung und Teilung von Macht ausgeht, befasst sich die accountability-Forschung mit der Frage, wer wen in welcher Weise mit welchen Kompetenzen kontrollieren sollte (siehe auch Lauth in diesem Band).

Vor diesem Hintergrund untersucht dieser Beitrag die Frage, gegenüber wem und in welcher Weise supranationale Organisationen im EU-Mehrebenensystem

\footnotetext{
${ }^{1}$ Für kritische Anregungen dankt die Autorin Timm Beichelt, Claudia Fahron, Miriam Hartlapp, Markus Gastinger, Laura Mahrenbach, Matthias Matthijs, Henning Schmidtke, Claudia Wiesner, den anonymen Gutachtern und den Teilnehmern der AKI-Tagung „Gewaltenteilung und Demokratie im Mehrebenensystem der EU“ am 9.-10.10.14 an der FU Berlin und der EUSA 2015. Für die sprachliche Editierung bedankt sich die Autorin bei Claudia Fahron und Markus Gastinger. Dieser Beitrag ist Teil des Projekts Delegation of Power to International Organizations and Institutional Empowerment over Time, dessen Förderung durch das European Union's seventh framework for research, technological development and demonstration, ERC Consolidator Grant No. 312368 ich hiermit dankbar anzeige.

${ }^{2}$ In Anlehnung an die Einleitung dieses Bandes wird Macht definiert als Einflussmöglichkeiten und Kompetenzen, die mit der Ausübung politischer Herrschaft verbunden sind und Entscheidungs- und Durchsetzungskompetenzen umfassen können.
} 
rechenschaftspflichtig sind. ${ }^{3}$ Aufbauend auf Erkenntnissen der accountability- und Delegationsliteratur (Alter 2008; Bovens 2010; Grant und Keohane 2005; Hawkins et al. 2006; Lauth 2007; Majone 2001) unterscheide ich zwei Modi der Verantwortungszurechnung in der EU-Governance: Delegations- und Treuhandmodi. Während die Prinzipal-Agenten-Delegationsbeziehung (PA) durch formale hierarchische, überwachende und rechtlich verbindliche Kontrollmechanismen charakterisiert ist, erfolgt Kontrolle und Verantwortungszurechnung im Treuhandmodus überwiegend informell durch gleichrangige Organisationen (peers) und öffentliche Reputationsmechanismen.

Der Beitrag ist wie folgt gegliedert. Abschnitt 2 stellt die zwei Modi der Verantwortungszurechnung im EU-Mehrebenensystem dar. Abschnitt 3 vergleicht die Kontrollmechanismen in diesen beiden Modi der accountability. Zur Illustration des Arguments wird ein Delegationsmodus (Abschn. 4) zwischen dem Rat der EU und der Kommission und ein Treuhandmodus zwischen den Eurostaaten und der EZB (Abschn. 5) bei der Umsetzung der Hilfsprogramme während der Eurokrise analysiert. Dabei greife ich auf Primärquellen - wie interne Unterlagen der Kommission, des Europäischen Parlamentes (EP), der EZB und des Rats der EU - sowie auf Interviews mit Beamtinnen dieser Organisationen zurück. ${ }^{4}$ Abschießend diskutiere ich die gewonnenen Erkenntnisse, zeige, welche Implikationen sich aus dem Vergleich dieser zwei Modi ergeben, und verweise auf die Grenzen der accountabilityPerspektive (Abschn. 6).

\section{Modi der accountability in der EU-Governance}

Governance in der EU ist durch die Verflechtung von Akteuren und Ebenen mit unterschiedlicher Kompetenzausstattung und die damit einhergehenden komplexen Aushandlungsprozesse charakterisiert (Benz 2009; Grande 2000). Dieses Mehrebenensystem umfasst drei Dimensionen der Trennung und Verschränkung von Macht: die innere Teilung, die horizontale Gewaltenteilung und die vertikale Dynamik zwischen den Ebenen. Dabei sticht das Regierungssystem der EU durch zwei Besonderheiten hervor. Zum einen ist Macht nicht eindeutig auf der nationalen oder auf der supranationalen Ebene verortet. Zum anderen kontrollieren EU-Institutionen Mitgliedstaaten bei der Implementierung gemeinschaftlicher Regeln (siehe die Einleitung zu diesem Band). Dies wirft Fragen der Legitimität, Kontrollpraktiken und Verantwortungszurechnung im EU-Mehrebenensystem auf. Insbesondere wenn speziell geschaffene informelle Gremien hinzutreten, wie z. B. die der TroikaInstitutionen, ist die Zuordnung der Verantwortlichkeit und der Kompetenz und somit die Möglichkeit der accountability erschwert.

\footnotetext{
${ }^{3} \mathrm{Da}$ sich diese Arbeit auf EU-Governance konzentriert, wird auf die Rolle des IWF nicht weiter eingegangen.

${ }^{4}$ Die Autorin hat im Februar und März 2014 Interviews mit insgesamt sechs Vertretern der TroikaInstitutionen in Lissabon durchgeführt. Dabei handelte es sich um teilstandardisierte Interviews, die zwischen 60 und 90 min dauerten. Die Auswahl der Interviewpartnerinnen erfolgte dadurch, dass diese die jeweiligen Institutionen in einem der Krisenländer vertraten und dort für die Überwachung der Umsetzung der Hilfsprogramme zuständig waren.
} 
Analog zur demokratietheoretischen Diskussion geht es in diesem Beitrag um die Bedeutung von Gewaltenteilung im Sinne der Machtbalance zwischen verschiedenen Organen und der Machtverschränkung zu Kontrollzwecken. Dabei steht die Frage im Mittelpunkt, wer in welcher Weise die Troika-Institutionen kontrolliert. Zur Beantwortung dieser Fragestellung greift dieser Beitrag auf den PA-Ansatz und accountability-Studien zurück, da sich beide Zugänge mit Fragen der Verantwortungszurechnung, bezogen auf die Verpflichtung eines Akteurs, sein Verhalten gegenüber einem anderen zu rechtfertigen (Bovens 2007, S. 450), befassen. Schedler (1999, S. 17) definiert zutreffend: „A schuldet B Rechenschaft, wenn A verpflichtet ist $B$ über seine (vergangenen oder zukünftigen) Handlungen zu informieren und sich für diese zu rechtfertigen sowie eine Bestrafung im Falle von Verfehlungen befürchten muss".

Während PA-Studien den Handlungsspielraum und die Autonomie des Agenten, Informationsasymmetrie, Kontrollmechanismen seitens der Prinzipale und Strategien der Agenten, dieser Kontrolle zu entkommen, untersuchen (siehe z. B. Bauer et al. 2015; Conceição-Heldt 2013; Grigorescu 2010; Hawkins et al. 2006; Lupia 2003; Pollack 2003), konzentrieren sich accountability-Studien auf normative oder soziale Mechanismen der Verantwortungszurechnung (Bovens 2010; Brandsma 2013; Grant und Keohane 2005; Lauth 2007). Auf der normativen Ebene beinhaltet accountability eine Reihe von Regeln oder Maßstäben zur Evaluation der Performanz von Agenten. Auf der Ebene der sozialen Mechanismen geht es um institutionelle Arrangements zwischen accountors und accountees (Bovens 2010, S. 947), bei denen Agenten oder Treuhänder von Dritten überwacht werden. Accountors werden hier verstanden als diejenigen Akteure (Agenten oder Treuhänder), die sich für ihre Handlungen rechtfertigen müssen. Accountees sind hingegen Akteure (Prinzipale oder Begünstigte), welche die accountors zur Rechenschaft ziehen können (siehe auch Pollitt 2003, S. 89).

Der Delegationsmodus ist dadurch gekennzeichnet, dass Agenten gegenüber kollektiven oder multiplen Prinzipalen oder sogar einem Dritten Rechenschaft ablegen müssen. So agiert die Kommission in der Troika-Konstellation als Agent des Europäischen Rats oder des Rats der EU, die als kollektive Prinzipale auftreten. In anderen institutionellen Konfigurationen, bei denen die Kommission im Auftrag des Rats der EU und des Europäischen Parlaments handelt, die sogenannte multiple Prinzipale bilden, ist die Kommission gegenüber Ministerrats- und Parlamentsausschüssen aber auch gegenüber Dritten, wie dem Europäische Gerichtshof (EuGH) oder dem Europäische Ombudsmann, rechenschaftspflichtig. Wenn ein Agent seine Kompetenzen überschreitet oder entgegen den Präferenzen der Prinzipale handelt, indem er von seinem Mandat abweicht (Conceição-Heldt 2013, S. 26), kann er theoretisch von seinen Prinzipalen oder durch Dritte sanktioniert werden. Allerdings erweist es sich in der Praxis als äußerst schwierig, dem Agenten agency slack nachzuweisen. Denn Prinzipale haben heterogene Präferenzen hinsichtlich des Handlungsspielraums eines Agenten bei der Ausführung des ihm auferlegten Mandats und können sich z. B. oftmals nicht darauf einigen, ob ein Agent seine Kompetenzen überschritten hat.

Im Unterschied zu PA-Studien befassen sich accountability-Studien mit drei Dimensionen von Verantwortungszurechnung: Information, Rechtfertigung und der 
Möglichkeit der Sanktionierung. Information bezieht sich darauf, dass accountors dazu angehalten sind, ihren acountees über ihre Entscheidungen zu berichten. Rechtfertigung bedeutet, dass accountability holders ihre accountors hinsichtlich der Angemessenheit ihrer Handlungen zur Rechenschaft ziehen können (Bovens et al. 2014, S. 9). Im Falle eines Machtmissbrauchs können accountees ihre accountors sanktionieren (Grant und Keohane 2005, S. 34; Schedler 1999, S. 14). ${ }^{5}$ Buiter (2007) unterscheidet ferner zwischen formaler und substantieller accountability. Formale accountability wird verstanden als ex post-Rechtfertigung. Diese Form der accountability setzt aber voraus, dass Prinzipale die Handlungen ihrer Agenten überwachen können. Letztere wiederum müssen ihren Prinzipalen ausreichende Informationen für ein fundiertes Urteil zur Verfügung stellen. Substantielle accountability bezieht sich hingegen darauf, dass nach der Berichterstattung und Rechtfertigung die Möglichkeit der Sanktionierung im Falle von Verfehlung gegeben ist. Buiter (2007) weist aber gleichzeitig darauf hin, dass Prinzipale oder Begünstigte, die sich für eine hohe Autonomie von Zentralbanken entscheiden, gleichzeitig ihre Kontrollmechanismen beschränken. Infolgedessen ist die dritte Dimension der accountability, nämlich die Auferlegung von Sanktionen bei einer Überschreitung der Kompetenzen oder des Mandats, im Treuhandmodus von vorneherein nicht gegeben.

Verantwortungszurechnung in der EU erfolgt nach dem Delegations- und Treuhandmodus. Im Delegationsmodus der accountability handeln supranationale Organisationen als Zweckagenten der sie ermächtigenden Prinzipale (Grant und Keohane 2005, S. 31 f.). Kontrollmechanismen sind hierarchisch, formal und zentralisiert - meistens in Form von Ausschüssen - und gehören zu den sogenannten „Polizeistreifen“-Typen (police patrol; McCubbins und Schwartz 1984). Im Treuhandmodus hingegen werden Treuhänder (trustees) als Ermessensautoritäten verstanden, die mit einem erheblichen Handlungsspielraum ausgestattet sind. Kontrollmechanismen in Form von „Feueralarm“ (fire alarm) sind informeller Natur und erfolgen durch Dritte (McCubbins und Schwartz 1984). Dies können Bürger, Interessenverbände, Nichtregierungsorganisationen, Parlamente oder Gerichte sein. In diesem Verantwortungszurechnungsmodus haben Begünstigte vollkommenes Vertrauen in die Fachexpertise ihrer Treuhänder. Diese Fachexpertise stellt gleichzeitig die Quelle ihrer Autorität dar und verleiht ihrem Handeln Legitimität. Treuhänder sind ferner dazu ermächtigt, nicht nur im Interesse der Begünstigten zu handeln, sondern auch Dritten (z. B. den EU-Bürgern) zu dienen (Alter 2008, S. 39 f.). Daraus folgt, dass bei Treuhandbeziehungen Ermächtigte ihr Amt nicht nur gewissenhaft auslegen sollen, sondern zur Einhaltung ihrer Sorgfaltspflicht (duty of care) im Mandat explizit angehalten werden. So ist es z. B. die Aufgabe der EZB, die Währungsstabilität zu wahren, und die des EuGH, die Einhaltung europäischer Verpflichtungen zu überwachen (Alter 2008, S. 39 f.). Im Gegensatz zum Delegationsmodus wird Unabhängigkeit als notwendige Bedingung zur Erfüllung der Treuhändermandate gesehen. Wenn Richter oder Zentralbanker zur Einhaltung ihrer Sorgfaltspflicht von den Präferenzen der Begünstigten abweichen, wird dies nicht als Machtmissbrauch gewertet (Grant und Keohane 2005, S. 32).

${ }^{5}$ Der Fokus dieses Beitrags liegt ausschließlich auf den beiden ersten Dimensionen. 
Diese beiden Modi der Verantwortungszurechnung unterscheiden sich somit durch die Beziehungen zwischen den Akteuren, die ermächtigten Institutionen, das erwartete Verhalten dieser Akteure und die verwendeten Kontrollmechanismen. Im nächsten Abschnitt gehe ich näher auf die in diesen zwei Modi der accountability verankerten Kontrollmechanismen ein.

\section{Kontrollmechanismen in Delegations- und Treuhandbeziehungen}

Delegations- und accountability-Studien operationalisieren Kontrollmechanismen in ähnlicher Weise, auch wenn sie hierfür unterschiedliche Konzepte verwenden. Während Grant und Keohane (2005, S. 35 ff.) sieben accountability-Mechanismen der Weltpolitik - hierarchische, überwachende, fiskale, rechtliche, marktwirtschaftliche, gleichrangige (peers) und öffentliche Reputation - identifizieren, unterscheidet Bovens (2007, S. 455 ff.) zwischen politischer, rechtlicher, administrativer, professioneller und sozialer Rechenschaftspflicht. ${ }^{6}$ In der PA-Literatur dagegen werden insbesondere fünf Kontrollmechanismen hervorgehoben: Selektionsprozeduren, Delegationsmandate, Überwachung und Berichtserfordernisse, institutionelle checks and balances sowie Sanktionen. Aufbauend auf diesen Studien geht es in diesem Beitrag um den Vergleich der zwei Modi der accountability hinsichtlich der darin verankerten Kontrollmechanismen: formale im Delegations- und informelle im Treuhandmodus. Diese wiederum können zu niedrigerer oder höherer Verantwortungszurechnung der Agenten/Treuhänder gegenüber ihren Prinzipalen/ Begünstigten führen.

Formale und zentralisierte Kontrollmechanismen beziehen sich auf ex ante- und ex post-Rechtfertigungen der Agenten. Diese Form der accountability ermöglicht es Prinzipalen, die Handlungen ihrer Agenten effektiv auf unterschiedlichen Ebenen zu überwachen. Sie kann folgende Kontrollmechanismen im Delegationsmodus umfassen: hierarchische, überwachende und rechtliche.

Hierarchische Mechanismen beziehen sich auf den Grad der Verantwortungszurechnung innerhalb der EU-Organe und setzen mehrere Ebenen voraus (Benz 2015; Bovens 2007; Grant und Keohane 2005). Verantwortungszurechnung beginnt an der Spitze einer Organisation mit den Präsidenten der EZB oder der Kommission und den Generaldirektoren als accountability holders und untergeordneten Funktionären als Ermächtigten (Grant und Keohane 2005, S. 36). Wenn Beamte sich nicht an die Anweisungen ihrer Vorgesetzten halten, kann dies zur Beeinträchtigung ihrer Karrierechancen bei der Organisation oder zur Einschränkung ihres Handlungsspielraumes führen.

Überwachende Kontrollmechanismen beschreiben Instrumente formalisierter oder informeller Art, bei denen sich die Präsidenten der EZB oder der Kommission gegenüber den EU-Mitgliedstaaten (beim Europäischen Rat oder dem Rat der EU)

\footnotetext{
${ }^{6}$ Accountability-Studien benutzen eher den Begriff Verantwortungszurechnungsmechanismen. In diesem Beitrag verwende ich hingegen, in Anlehnung an Delegationsstudien, das Konzept der Kontrollmechanismen.
} 
Tab. 1 Modi der Verantwortungszurechnung im EU-Mehrebenensystem. (Quelle: Eigene Darstellung basierend auf Grant und Keohane (2005) sowie Bovens (2007))

\begin{tabular}{|c|c|c|c|c|c|}
\hline & Kontrollmec & anismen & $\begin{array}{l}\text { Accountability } \\
\text { holders }\end{array}$ & Accountors & $\begin{array}{l}\text { Kosten für } \\
\text { accountors }\end{array}$ \\
\hline \multirow[t]{3}{*}{$\begin{array}{l}\text { Delegations- } \\
\text { modus }\end{array}$} & \multirow[t]{3}{*}{$\begin{array}{l}\text { formal und } \\
\text { zentralisiert }\end{array}$} & Hierarchische & $\begin{array}{l}\text { Präsidenten } \\
\text { der EZB } \\
\text { oder der } \\
\text { Kommission }\end{array}$ & $\begin{array}{l}\text { Untergeordnete } \\
\text { Funktionäre } \\
\text { (Kommissare, } \\
\text { General- } \\
\text { direktoren, } \\
\text { Beamte) }\end{array}$ & $\begin{array}{l}\text { Beein- } \\
\text { trächtigung der } \\
\text { Karriereaussichten }\end{array}$ \\
\hline & & Überwachende & Staaten & $\begin{array}{l}\text { Präsidenten der } \\
\text { EZB oder der } \\
\text { Kommission }\end{array}$ & $\begin{array}{l}\text { Einschränkung des } \\
\text { Handlungsspiel- } \\
\text { raumes; Verlust des } \\
\text { Amtes }\end{array}$ \\
\hline & & Rechtliche & EuGH & $\begin{array}{l}\text { EZB oder } \\
\text { Kommission; } \\
\text { Mitgliedstaaten }\end{array}$ & $\begin{array}{l}\text { Verlust an Autorität; } \\
\text { Beauftragung eines } \\
\text { neuen Agenten }\end{array}$ \\
\hline \multirow[t]{2}{*}{$\begin{array}{l}\text { Treuhand- } \\
\text { modus }\end{array}$} & \multirow[t]{2}{*}{$\begin{array}{l}\text { informell } \\
\text { und de- } \\
\text { zentralisiert }\end{array}$} & $\begin{array}{l}\text { Peer } \\
\text { Organisationen }\end{array}$ & $\begin{array}{l}\text { EP, } \\
\text { Europäischer } \\
\text { Ombudsmann }\end{array}$ & $\begin{array}{l}\text { Präsidenten der } \\
\text { EZB oder der } \\
\text { Kommission }\end{array}$ & $\begin{array}{l}\text { Auswirkungen auf } \\
\text { Netzwerke; Verlust } \\
\text { der Unterstützung } \\
\text { Anderer }\end{array}$ \\
\hline & & $\begin{array}{l}\text { Öffentlicher } \\
\text { Reputations- } \\
\text { verlust }\end{array}$ & $\begin{array}{l}\text { Öffentliche } \\
\text { Meinung }\end{array}$ & $\begin{array}{l}\text { Präsidenten der } \\
\text { EZB oder der } \\
\text { Kommission }\end{array}$ & $\begin{array}{l}\text { Diffuse } \\
\text { Reputationskosten; } \\
\text { Prestige }\end{array}$ \\
\hline
\end{tabular}

oder anderen supranationalen Organisationen, wie dem EP, für ihr Handeln rechtfertigen müssen.

Bei Rechtskontrollinstrumenten geht es um die Frage, ob das Handeln supranationaler Organisationen der vorgegebenen Rechtslage und ihrer Kompetenzzuweisung entspricht. In diesem Fall agiert der EuGH als Überwacher der anderen EU-Organe und auch der Mitgliedstaaten (vgl. dazu auch Kneip in diesem Band). Wenn der EuGH ein Fehlverhalten feststellt, z. B. wenn ein anderes EU-Organ seine Kompetenzen überschritten hat, dann kann theoretisch die Sanktionsgewalt der Mitgliedstaaten dazu führen, dass die materiellen Ressourcen eines Ermächtigten gekürzt werden (Budget oder Personal), seine Autorität beeinträchtigt wird und die Mitgliedstaaten sich sogar trotz der damit verbundenen Kosten für die Gründung einer alternativen Institution entscheiden (siehe Tab. 1).

Im Gegensatz dazu überwiegen im Treuhandmodus informelle und dezentralisierte Kontrollmechanismen durch peers oder öffentlichen Reputationsverlust. Buiter (2007) zeigt, dass formale Verantwortungszurechnung alle drei Elemente der Kontrolle (Information, Erklärung, Rechtfertigung) beinhaltet. Bei der substantiellen Verantwortungszurechnung hingegen verfügen Begünstigte nicht über wirksame Kontrollmechanismen, wie z. B. den Entzug des Delegationsmandats, wenn sie mit dem Handeln des Treuhänders unzufrieden sind.

Peer Kontrollmechanismen beziehen sich auf Instrumente der gegenseitigen Evaluation, bei denen supranationale Organisationen der Kontrolle anderer (gleichrangiger) Organisationen unterstehen (Bovens et al. 2014, S. 11). In der EU bedeutet dies, dass der EuGH als Judikative Kontrollaufgaben gegenüber den anderen EUInstitutionen wahrnimmt. Ferner kann das EP sowohl die Tätigkeit der Kommission als auch die der EZB überwachen. Der Europäische Rechnungshof und der Ombuds- 
mann fungieren im Allgemeinen auch als Kontrollinstanzen im EU-Mehrebenensystem. Bezugnehmend auf EuGH-Urteile hat das EP lange Zeit versucht, seine Kompetenz- und Kontrollfunktionen, insbesondere bei den EU-Agenturen und bei der Europäischen Kommission, auszudehnen (Lord 2011). Die Machtverschränkung der EZB durch peers ist hingegen nur auf der Berichterstattungsebene möglich. Da sich die Eurostaaten für ein institutionelles Design entschieden haben, das der EZB weitgehend operationale Unabhängigkeit gewährt, ist die EZB nur gegenüber dem EP, der Kommission und dem Europäischen Rat hinsichtlich des ersten Elements der Kontrolle (Berichterstattung) rechenschaftspflichtig.

Der zweite Kontrollmechanismus, öffentlicher Reputationsverlust, bezeichnet eine noch diffusere Form der Verantwortungszurechnung. Diese bezieht sich auf die Glaubwürdigkeit einer Institution, die auch Teil der formalen Kontrollmechanismen ist. Wenn es beispielsweise zur Evaluation des Handelns der Treuhänder kommt, berücksichtigen sowohl Begünstigte als auch peers die Reputation des Treuhänders (Grant und Keohane 2005, S. 37). Nicht nur die Öffentlichkeit, sondern auch die Glaubwürdigkeit unter den peers stellen wichtige Reputationsmechanismen dar (Majone 2001). Im Falle einer negativen Evaluation oder wenn eine Institution, wie z. B. die EZB, im Rahmen des Eurokrisenmanagements ins Kreuzfeuer der Öffentlichkeit oder einzelner Mitgliedstaaten gerät, riskiert sie diffuse Reputationsverluste sowie weniger Unterstützung durch peers oder einzelne Eurostaaten. Dies kann in letzter Instanz zu Prestige- und Glaubwürdigkeitsverlusten führen (siehe Tab. 1 und Abschn. 5 dieses Beitrags).

Dabei sollte nicht aus dem Blick verloren werden, dass diese formalen und informellen Kontrollmechanismen sich nicht gegenseitig ausschließen und sogar parallel zueinander zur Geltung kommen können. Einerseits können im Delegationsmodus Prinzipale zusätzlich zu hierarchischen und überwachenden auch auf rechtliche Kontrollmechanismen zurückgreifen, sofern sie sich darauf einigen können, dass der Agent außerhalb seines Delegationsmandats agiert hat. Andererseits können im Treuhandmodus peer und öffentlicher Reputationsverlust gleichzeitig auftreten.

Der nächste Abschnitt beschäftigt sich nun empirisch mit der Frage, gegenüber wem und in welcher Weise die Kommission und die EZB während der Staatsschuldenkrise rechenschaftspflichtig waren.

\section{Formale Kontrollmechanismen im Delegationsmodus}

Im Rahmen der Euro-Rettungsprogramme wurde die Kommission zusammen mit dem IWF und der EZB damit beauftragt, die Umsetzung der Programme in den Krisenländer zu überwachen. Die Mitgliedstaaten delegierten somit die Kompetenz, makroökonomische, fiskale, finanzmarkttechnische und strukturelle Reformen zu planen und umzusetzen, an die Kommission (Council of the European Union 2010; General Secretariat of the Council 2011).

Nach der Übertragung von Kompetenzen geht es im Sinne der Machtbeschränkung um die Kontrollmöglichkeiten seitens der Prinzipale. Wenn wir Schedlers (1999) Definition von Verantwortungszurechnung nehmen, dann ist das erste Element der Kontrolle die Bereitstellung von Information, oder konkret in diesem Fall, die schrift- 
liche Berichterstattung. Die zweite Komponente befasst sich mit dem Erklären und Rechtfertigen und somit mit der Überwachung des Agenten seitens der Prinzipale in formalisierten, ministeriellen Gremien auf der EU-Ebene. Auf der nationalen Ebene erfolgten in unterschiedlicher Weise Beschränkungen der Macht der TroikaInstitutionen durch die nationalen Parlamente.

Die Berichterstattung der Kommission erfolgte im Anschluss an die Überprüfung der Umsetzung der Nothilfeprogramme. Die Kommission erstellte die Fortschrittsberichte über den Grad der Implementierung und gesamtwirtschaftliche Entwicklungen in den betroffenen Ländern (Interview mit einem Kommissionsmitarbeiter aus der Troika-Delegation). Somit informierte sie die Eurostaaten umfassend über ihre Arbeit und über den Stand der Umsetzung der vierteljährlichen Compliance-Berichte seitens der Nehmerländer. Diese Berichte wurden nachfolgend in verschiedenen Ratsausschüssen präsentiert. Abhängig davon, welcher Kontrollmechanismus bei der Gewährung von Finanzhilfen zur Anwendung kam, berichtete die Kommission dem Wirtschafts- und Finanzausschuss oder der Arbeitsgruppe Eurogruppe im Rat der EU über Gestaltung, Verhandlung und Umsetzung der Finanzhilfsprogramme. Im nächsten Schritt leiteten die Vorsitzenden dieser Ausschüsse diese Information an die einzelnen Mitglieder weiter. Diese Kommissionsberichte bildeten die Grundlage für die Entscheidung seitens der Eurogruppe über die Überweisung der nächsten Hilfstranche an die betroffenen Eurokrisen-Länder (European Parliament 2014).

Die zweite Dimension der Verantwortungszurechnung, die Rechtfertigung, erfolgte durch überwachende und hierarchische Kontrollmechanismen. Überwachende accountability fand durch formalisierte, ministerielle Gremien auf der Ebene des Ministerratstreffens für Finanzen und Wirtschaft (ECOFIN) und der Eurogruppe, die aus den Finanzministern der Eurostaaten besteht, statt. Darüber hinaus war die Kommission gegenüber der Eurogruppe rechenschaftspflichtig (Interview mit einem Kommissionsmitarbeiter aus der Troika-Delegation). Dabei gab es folgende Arbeitsteilung: Rechtlich war ECOFIN für alle Angelegenheiten im Zusammenhang mit dem Europäischen Finanzstabilisierungsmechanismus (EFSM) verantwortlich, während die Eurogruppe zusammen mit den garantiegebenden Eurostaaten für die Belange der Europäischen Finanzstabilisierungsfazilität (EFSF) zuständig war (Eurogroup 2014). Schließlich übernahm das Direktorium des Europäischen Stabilisierungsmechanismus (ESM) als neu eingerichtete intergouvernementale Institution die Verantwortung für die Finanzhilfe (European Stability Mechanism 2014), die sich auf Kreditauflagen und makroökonomische Reformprogramme bezog, deren Einhaltung wiederum von der Kommission überwacht wurde (Committee on Economic und Monetary Affairs 2014).

Gleichzeitig kamen hierarchische Kontrollmechanismen zur Geltung, da die Kommissionsbeamten regelmäßig Bericht an die Generaldirektoren in Brüssel und den für die Nothilfeprogramme verantwortlichen Vize-Präsidenten der Kommission erstatteten. Ähnlich wie die anderen Troika-Institutionen entsandte die Kommission zahlreiche Mitarbeiter, in der Regel Ökonomen aus der Generaldirektion Wirtschaftsund Finanzfragen, in die betroffenen Länder. Diese Kommissionsmitarbeiter informierten regelmäßig ihre Generaldirektoren oder Referatsleiter. Durch diese Verstärkung der Personalressourcen durch Mitarbeiter der Generaldirektion Wirtschaftsund Finanzfragen, die in die Krisenstaaten entsandt wurden und wiederum an die 
Kommission in Brüssel zurück berichteten, waren mehrfach hierarchische Kontrollmechanismen vorhanden. Darüber hinaus wurde diese Information auch an andere beteiligte Generaldirektionen - wie z. B. Gesundheits-, Kohäsions-, Bildungs-, Energie-, Umwelt- und Sozialpolitik - weitergeleitet (Interviews mit Kommissionsund IWF-Mitarbeitern aus der Troika-Delegation).

Diese Kombination von hierarchischen und überwachenden Kontrollmechanismen erlaubte es den Eurostaaten, die Kommission in Form formalisierter, ministerieller Gremien zur Rechenschaft zu ziehen. Die kontinuierliche Berichterstattung erhöhte die Effektivität dieser Mechanismen. Die Verfügbarkeit ausführlicher und regelmäßiger Handlungsangaben sowie die Möglichkeit, ihren Agenten während der Umsetzung zu befragen, verringerte somit die Wahrscheinlichkeit von Verantwortungszurechnungsdefiziten.

Ferner erfolgte die Verantwortungszurechnung durch Berichterstattung und Zustimmung zu den einzelnen Teilen der Vereinbarung durch dienationalen Parlamente. Einige Beiträge dieses Bandes zeigen, dass der Deutsche Bundestag neue Kontrollund Entscheidungskompetenzen hinzugewonnen hat (siehe Abels, Buzogany/Kropp, Kneip, Wimmel und Wiesner), während die Parlamente der Empfängerstaaten in ihren Entscheidungskompetenzen noch weiter beschränkt wurden (siehe Wiesner). In Irland waren z. B. Vertreter des Parlaments an der Aushandlung des Memorandum of Understanding (MoU) mit den Troika-Institutionen nicht beteiligt. Kontrollmöglichkeiten ergaben sich nur hinsichtlich der finanziellen und gesetzgebenden Aspekte des $\mathrm{MoU}$ in Form von Informationsrechten und den finalen Umsetzungsberichten nach dem Abschluss der Verhandlungen mit den Troika-Institutionen, da das Parlament diesen zustimmen musste (European Parliament 2014). Auf der europäischen Ebene versuchte das EP direkte Kontrollmöglichkeiten über die Kommission zu erlangen, indem es ab Januar 2014 vier Missionen zur Evaluierung der Arbeit der Troika in die Krisenländer sendete. Dabei führten die Abgeordneten Gespräche mit Regierungsund Oppositionsvertretern, Sozialpartnern und den Zentralbanken. Treffen mit den Vertretern der Troika-Institutionen fanden hingegen nicht statt, weil das EP in den Augen der Troika-Vertreter in Wirtschafts- und Finanzfragen nicht mit den nötigen Mitentscheidungskompetenzen ausgestattet sei (Interview mit Vertretern der Kommission und des IWF). Zur Vorbereitung eines Berichts über die Evaluierung der Auswirkung der Hilfsprogramme schickte das EP einen Fragenkatalog an die vier Krisenländer, in dem es u. a. um die Rolle der nationalen Parlamente und der Sozialpartner bei der Aushandlung der Hilfsprogramme, des Handlungsspielraums bei den Konsolidierungs- und Strukturreformen und der Zusammenarbeit zwischen den Troika-Institutionen und den Nationalregierungen ging. Darüber hinaus erhielten der Vorsitzende der Eurogruppe, die Kommission und die EZB einen umfangreichen Fragenkatalog mit insgesamt 29 Fragen über das Design der Finanzhilfsprogramme sowie die Rolle der Troika bei der Umsetzung derselben. Diese Fragen bezogen sich auf accountability und Legitimität der Troika-Institutionen, die Position der einzelnen Mitgliedstaaten bei der Verabschiedung der Hilfsprogramme und die Arbeitsteilung zwischen den drei Institutionen (European Parliament 2014). 


\section{Informelle Kontrollmechanismen im Treuhandmodus}

Im Verlauf der Finanzhilfsprogramme bestand die Rolle der EZB grundsätzlich darin, die Bankensysteme der Krisenländer mit ausreichend Liquidität zu versorgen. Im Gegensatz zur Kommission und dem IWF übernahm die EZB eher eine beratende Rolle gegenüber den Nationalbanken in den Programmländern (Interview mit EZBMitarbeitern aus der Troika-Delegation). Auch hier stellt sich die Frage, gegenüber wem und in welcher Weise die EZB in der institutionellen Troika-Konstellation rechenschaftspflichtig war.

Wenn wir Schedlers (1999) Definition von Verantwortungszurechnung nehmen, ist deren erste Komponente die Verpflichtung der EZB, relevante Informationen zu ihrem Handeln im Zusammenhang mit der Wahrung der Preisstabilität offenzulegen. Die zweite Komponente besteht aus der Befragung der EZB in einem Forum, in dem sie sich für die Angemessenheit ihres Handelns rechtfertigen muss. Die Treuhandlogik setzt voraus, dass trustees als souveräne Entscheidungsträger mit großem Ermessensspielraum und mit großer Unabhängigkeit ausgestattet sind (vgl. auch Majone 2001). Die Gründung der EZB 1998 erfolgte nach dem Vorbild der Deutschen Bundesbank. Nach der Erfahrung mit der Hyperinflation der Weimarer Republik bevorzugte Deutschland in der Nachkriegszeit eine Geldpolitik, die zuverlässige Preisstabilität sicherstellte. Dies führte dazu, dass der Deutschen Bundesbank große Unabhängigkeit gewährt wurde, um zu verhindern, dass Geldpolitik von fiskalpolitischen Überlegungen geleitet wurde. Im Rahmen der Verhandlungen zum Maastricht-Vertrag, und somit zur Wirtschafts- und Währungsunion, war es vor allem Deutschland wichtig, eine strikte Trennung zwischen Geld- und Fiskalpolitik zu haben. Infolgedessen stimmte Deutschland einer europäischen Bank zu, die nach dem Vorbild der Bundesbank mit großer Unabhängigkeit gegenüber den nationalen Regierungen ausgestattet wurde (Heisenberg und Richmond 2011).

Mit der Gründung der EZB übertrugen die Mitgliedstaaten umfassende Entscheidungskompetenzen an eine supranationale Institution, womit die EZB gemäß ihrer Statuten und Art. 282 des Vertrags über die Arbeitsweise der EU bei der Ausübung ihrer Befugnisse über weitreichende Unabhängigkeit hinsichtlich einer Einmischung der Eurostaaten und der anderen supranationalen Organisationen verfügt (siehe auch Elgie 2002). Zusätzlich ist die EZB auch finanziell autonom, da ihr Budget und Stammkapital ausschließlich von den nationalen Notenbanken stammt. Diese institutionalisierte Unabhängigkeit wird weiterhin durch die lange und begrenzte Amtszeit von Direktoriumsmitgliedern (acht Jahre, keine Wiederernennung zulässig), die Geheimhaltung und Einstimmigkeit bei der Entscheidungsfindung sowie restriktive Amtsenthebungsverfahren gestärkt (Heisenberg und Richmond 2011, S. 209).

Die hohe operative Unabhängigkeit der EZB ging mit einer geringen Verantwortungszurechnung einher. Formale Kontrollmechanismen durch peers und durch die Öffentlichkeit sind somit die einzigen Mechanismen der Machtverschränkung. Die EZB ist dazu verpflichtet, den anderen EU-Organen (dem Europäischen Rat, dem Rat der EU, dem EP und der Kommission) wöchentliche Finanzaufstellungen sowie vierteljährliche und jährliche Berichte über ihre Aktivitäten im Eurosystem und über die Geldpolitik für das letzte und laufende Jahr vorzulegen (European Central Bank 
2014). Dabei bleibt es in ihrem Ermessen zu entscheiden, wie umfangreich und detailliert diese Berichterstattung erfolgt. Die Eurostaaten verfügen über keinerlei Möglichkeiten, das Handeln der EZB zu beeinflussen oder zu sanktionieren, wenn diese ihre Handlungskompetenzen überschreitet. Verantwortungszurechnung erfolgt durch Dritte (Bürger oder Gerichtshöfe) oder durch die Öffentlichkeit.

Im Verlauf der Eurokrise gab es verschiedene Versuche die Macht der EZB zu verschränken. Als der Rat der EZB am 6. September 2012 einen Beschluss über „Technical Features of Outright Monetary Transactions“ (OMT) fasste, in dem vorgesehen war, dass die EZB Staatsanleihen ausgewählter Eurostaaten in unbegrenzter Höhe ankaufen könne, führte diese Entscheidung zur Auslösung eines Feueralarmkontrollmechanismus in Form Dritter (Bürgerverein und Gerichtshöfe). Unter der Leitung einer Gruppe von Professoren und des CSU-Bundestagsabgeordneten Peter Gauweiler klagte eine Gruppe von 37.000 deutschen Bürgern, vertreten im Verein „Mehr Demokratie“, gegen diesen OMT-Beschluss vor dem deutschen Bundesverfassungsgericht, weil die EZB somit über ihr Mandat hinausgegangen sein und gegen das Verbot monetärer Haushaltsfinanzierung verstoßen habe (vgl. Kneip in diesem Band). In seiner Stellungnahme im Februar 2014 legte das Bundesverfassungsgericht zwar fest, dass das Ankaufprogramm von Staatsanleihen eine „ersichtliche Kompetenzüberschreitung" der EZB darstelle, verwies aber gleichzeitig wegen der rechtspolitischen Dimension des Vorgangs auf den EuGH, der über die Rechtmäßigkeit des OMT-Programms entscheiden sollte (Bundesverfassungsgericht 2014). Auf Vorlage des Bundesverfassungsgerichts entschied der EuGH, dass die EZB im Rahmen ihres Mandats gehandelt habe, denn das OMT-Programm gehöre zum Bereich der Währungspolitik und falle somit unter die Befugnisse der EZB (Court of Justice of the European Union 2015). Verantwortungszurechnung erfolgte somit durch Feueralarmmechanismen. Zwar gab es formale Ausformungen der Kontrolle, in der Praxis war aber durch den hohen Grad der operationalen Unabhängigkeit die Verschränkung der Macht und somit die Verantwortungszurechnung der EZB gegenüber Dritten gering.

In der Troika-Konstellation erfolgte Verantwortungszurechnung durch informelle Kontrollmechanismen in Form von gleichrangigen Organisationen und öffentlichem Reputationsverlust. Accountability gegenüber anderen peers geschah auf einer informellen Ebene in Form von regelmäßigen Berichten an die Eurogruppe und den Ausschuss für Wirtschafts- und Währungsfragen des EPs. Entweder der EZBPräsident oder andere Direktoriumsmitglieder standen dem EP dort Rede und Antwort. Die EZB begleitete die Verhandlung, Einrichtung und Implementierung der Nothilfeprogramme mit Rat und Expertise bezüglich der Geldpolitik und Wahrung der Preisstabilität. Zusammen mit der Kommission bewertete die EZB gegenüber den Euro-Finanzministern vierteljährlich die Rahmenbedingungen der Wirtschaftspolitik und die Einhaltung der Bedingungen, die mit der Gewährung von Finanzhilfe einhergingen und deren Verlängerung beeinflussten (European Parliament 2014).

Ähnlich wie die Kommission erhielt die EZB einen umfangreichen Fragenkatalog über ihre Rolle bei der Umsetzung der Staatshilfsprogramme. Die Antworten der EZB waren minimalistischer Natur. Sie berief sich z. B. darauf, dass sie durch ihre Fachexpertise zur Finanzstabilität beitrage und zusammen mit der Kommission die Umsetzung der an die Staatshilfsprogramme geknüpften Bedingungen überprüfe 
(European Parliament 2014). Das EP unternahm somit Versuche der Kontrolle und der Verantwortungszurechnung der EZB zusätzlich zu den vorgesehenen Anhörungen im Rahmen der Berichterstattung im Wirtschafts- und Finanzausschuss des EPs, ist dabei aber durch ähnliche Faktoren begrenzt, wie im Bereich der Haushaltspolitik.

Weitere Verantwortungszurechnung erfolgte durch die mediale Öffentlichkeit in Form von Pressemitteilungen und der Präsentation von Berichten (vgl. Interview mit EZB-Mitarbeitern aus der Troika-Delegation). Auch wenn es sich hier um eine minimalistische Berichterstattung aufgrund der operationalen Unabhängigkeit der EZB handelte, wurde die EZB in der deutschen Öffentlichkeit, insbesondere durch das OMT-Programm und die Einführung eines negativen Zinssatzes für Einlagen von Geschäftsbanken, heftig kritisiert (siehe hierzu z. B. die Berichterstattung in der Frankfurter Allgemeinen Zeitung und im Tagesspiegel).

Auch wenn die EZB nach dem Vorbild der Deutschen Bundesbank mit hoher Autonomie gegenüber der Politik ausgestattet wurde, kam es insbesondere durch den EZBAnkauf von Staatsanleihen finanzschwacher Eurostaaten seit Beginn der Eurokrise zu vermehrter Kritik durch Vertreter Deutschlands im Rat der EZB. Nacheinander schieden Axel Weber und Jürgen Starck im Februar und im September 2011 aus dem Rat der EZB aus. Zwar war Deutschland, zusammen mit zwei anderen Zentralbankpräsidenten, gegen die Wiederaufnahme des Ankaufprogramms von Staatsanleihen, wurde aber von den 23 anderen Ratsmitgliedern überstimmt. Im Dezember 2013 folgte dann der Rücktritt Jörg Asmussens. Das Ausmaß des Vertrauens in die EZB und in deren Fachexpertise war somit seitens Deutschlands beeinträchtigt.

\section{Fazit}

Gewaltenteilung setzt Kontrolle und Verschränkung von Macht voraus. In diesem Beitrag ging es um die Frage, gegenüber wem und in welcher Weise supranationale Organisationen im Mehrebenensystem der EU während der Staatsschuldenkrise rechenschaftspflichtig waren und welche Bedingungen für horizontale und vertikale Gewaltenteilung greifen. Dabei wurden der Delegations- und Treuhandmodus der Verantwortungszurechnung im Hinblick auf die darin verankerten Kontrollmechanismen miteinander verglichen. Dieser Vergleich zeigte, dass vielfältige Kontrollmechanismen formaler und informeller Natur der Machtverschränkung existierten, die zu höherer oder niedrigerer Fähigkeit der Kontrolle der Troika-Institutionen führten. In der Delegationsbeziehung überwogen hierarchische und überwachende Kontrollmechanismen durch formalisierte, ministerielle Gremien auf unterschiedlichen europäischen Ebenen (ECOFIN, Eurogruppe, Arbeitsgruppe Eurogruppe). Die Treuhandbeziehung hingegen war durch informelle Kontrollmechanismen der peer Organisationen und öffentlichen Meinung geprägt.

Dieser Beitrag verdeutlicht auch die Grenzen der accountability Perspektive. Während im Delegationsmodus vielfältige formale Kontrollmechanismen existierten, war im Treuhandmodus die Unabhängigkeit ausschlaggebend. Dies führte wiederum dazu, dass weder die Verantwortungszurechnung noch die Verschränkung der Macht der EZB zentral waren. Der EZB-Fall zeigt aber auch: Je höher der Grad der operationalen Unabhängigkeit, desto geringer die substantielle Verantwortungs- 
zurechnung einer supranationalen Organisation. Die EZB wurde nach dem Vorbild der Deutschen Bundesbank mit hoher Autonomie gegenüber den Eurostaaten ausgestattet. Auch wenn Treuhandbeziehungen theoretisch durch volles Vertrauen in die Fachexpertise der Treuhänder charakterisiert sind, hat in der Praxis das Ausmaß an Vertrauen in die Fachexpertise seitens einiger Eurostaaten abgenommen, da auf Maßnahmen wie das OMT-Programm zurückgegriffen wurde, die nicht durch alle Eurostaaten unterstützt wurden. Im Gegensatz zum Delegationsmodus, bei dem Agenten durch Prinzipale entlassen und sanktioniert werden können, ist dies im Treuhandmodus nicht möglich.

Open Access Dieser Artikel unterliegt den Bedingungen der Creative Commons Attribution License. Dadurch sind die Nutzung, Verteilung und Reproduktion erlaubt, sofern der/die Originalautor/en und die Quelle angegeben sind.

\section{Literatur}

Alter, Karen J. 2008. Agents or trustees? International courts in their political context. European Journal of International Relations 14 (1): 33-63.

Bauer, Michael W., Eugénia da Conceição-Heldt, und Jörn Ege. 2015. Autonomiekonzeptionen internationaler Organisationen im Vergleich. PVS Sonderheft Internationale Organisationen 49: 28-53.

Benz, Arthur. 2009. Politik im Mehrebenensystem. Wiesbaden: VS Springer.

Benz, Arthur. 2015. European public administration as multilevel system administration - a conceptual framework. In: The palgrave handbook of the European administrative system, Hrsg. Michael W. Bauer und Jarle Trondal, 42-65. Basingstoke: Palgrave Macmillan.

Bovens, Mark. 2007. Analysing and assessing accountability: A conceptual framework. European Law Journal 13 (4): 447-468.

Bovens, Mark. 2010. Two concepts of accountability: Accountability as a virtue and as a mechanism. West European Politics 33 (5): 947-967.

Bovens, Mark, Thomas Schillemans, und Robert E. Goodin. 2014. Public Accountability. In Oxford handbok of public accountability Hrsg. Mark Bovens, Thomas Schillemans und Robert E. Goodin, 1-24. Oxford: Oxford University Press.

Brandsma, Gijs. 2013. Controlling comitology: Accountability in a multi-level system, Basingstoke: Palgrave Macmillan.

Buiter, Willem H. 2007. Central Banks as Economic Institutions. Paris: Cournot Centre for Economic Studies.

Bundesverfassungsgericht. 2014. Hauptsacheverfahren ESM/EZB: Urteilsverkündung sowie Vorlage an den Gerichtshof der Europäischen Union. Bundesverfassungsgericht: Pressemitteilung Nr. 9/2014 vom 7. Februar 2014, Karlsruhe, https://www.bundesverfassungsgericht.de/SharedDocs/Pressemitteilungen/DE/2014/bvg14-009.html. Zugegriffen: 6. Juli 2015.

Committee on Economic and Monetary Affairs. 2014. Report on the enquiry on the role and operations of the Troika (ECB, Commission and IMF) with regard to the euro area programme countries. Brüssel: Committee on Economic and Monetary Affairs.

Conceição-Heldt, Eugénia da. 2013. Do agents „Run Amok“? Agency slack in the EU and US trade policy in the Doha round. Journal of Comparative Policy Analysis 15 (1): 21-36.

Council of the European Union. 2010. Statement by the heads of state and government of the euro area, 25 März 2010. Brüssel: Council of the European Union.

Court of Justice of the European Union. 2015. The OMT Programme Announced by the ECB in September 2012 is Compatible with EU Law Luxembourg. Vol. Press Release No 70/15, Judgement in Case C-62/14 Gauweiler and Others.

Elgie, Robert. 2002. The politics of the European Central Bank: Principal-agent theory and the democratic deficit. Journal of European Public Policy 9 (2): 186-200.

Eurogroup. 2014. Eurogroup 2014. http://eurozone.europa.eu/eurogroup/. Zugegriffen: 24. März 2014. 
European Central Bank. 2014. Accountability. Frankfurt. https://www.ecb.europa.eu/ecb/orga/ accountability/html/index.en.html. Zugegriffen: 3. Okt. 2014.

European Parliament. 2014. ECB's replies to the questionnaire of the European Parliament supporting the own initiative report evaluating the structure, the role and operations of the ,Troika '(Commission, $E C B$ and the IMF) actions in euro area programme countries. Brüssel: European Parliament.

European Stability Mechanism. 2014. Scope of Activity. Luxemburg, http://www.esm.europa.eu/. Zugegriffen: 24. März 2014.

General Secretariat of the Council. 2011. The European Council in 2010. Luxemburg: Publications Office of the European Union.

Grande, Edgar. 2000. ,Multi-Level Governance: Institutionelle Besonderheiten und Funktionsbedingungen des europäischen Mehrebenensystems'. In Wie Problemlösungsfähig ist die EU? Regieren im europäischen Mehrebenensystem, Hrsg. E. Grande, und M. Jachtenfuchs, 11-30. Baden-Baden: Nomos.

Grant, Ruth W., und Robert O. Keohane. 2005. Accountability and abuses of power in world politics. American Political Science Review 99 (1): 29-43.

Grigorescu, Alexandru. 2010. The Spread of Bureaucratic Oversight Mechanisms across Intergovernmental Organizations. International Studies Quarterly 54 (3): 871-886.

Hawkins, Darren G., David A. Lake, Daniel L. Nielson, und Michael J. Tierney. 2006. Delegation under anarchy: States, international organizations, and principal-agent theory. In Delegation and agency in international organizations, Hrsg. Darren G. Hawkins, David A. Lake, Daniel L. Nielson und Michael J. Tierney, 3-38. Cambridge: Cambridge University Press.

Heisenberg, Dorothee, und Amy Richmond. 2011. Supranational institution-building in the European Union: A comparison of the European Court of Justice and the European Central Bank. Journal of European Public Policy 9 (2): 201-218.

Lauth, Hans-Joachim. 2007. „Horizontal accountability“. Aktuelle Aspekte der Gewaltenteilung: Ein Vorschlag zur Systematisierung der Kontrollfunktion der Gewaltenteilung. In Gewaltenteilung und Demokratie. Konzepte und Probleme der ,horizontalen accountability “im interregionalen Vergleich, Hrsg. Sabine Kropp und Hans-Joachim Lauth, 45-71. Baden-Baden: Nomos Verl.-Ges.

Lord, Christopher. 2011. The European parliament and the legitimation of agencification. Journal of European Public Policy 18 (6): 909-925.

Lupia, Arthur. 2003. Delegation and its perils. In Delegation and accountability in parliamentary democracies, Hrsg. Kaare Strom, Wolfgang C. Müller und Torbjörn Bergman, 33-54. Oxford: Oxford University Press.

Majone, Giandomenico. 2001. Two logics of delegation: Agency and fiduciary relations in EU governance. European Union Politics 2 (1): 103-122.

McCubbins, Mathew D., und Thomas Schwartz. 1984. Congressional oversight overlooked: Police patrols versus fire alarms. American Journal of Political Science 28 (1): 165-179.

Pollack, Mark A. 2003. The engines of European integration: Delegation, agency, and agenda setting in the EU. Oxford: Oxford University Press.

Pollitt, Christopher. 2003. The essential public manager. Berkshire: Open University Press.

Schedler, Andreas. 1999. Conceptualizing accountability. In The self-restraining state: Power and accountability in new democracies, Hrsg. Andreas Schedler, Larry Diamond und Marc F. Plattner, 13-28. Boulder: Lynne Rienner Pub. 